\title{
A CHARACTERIZATION OF NONNEGATIVE BOX-GREEDY MATRICES *
}

\author{
ULRICH FAIGLE ${ }^{\dagger}$, ALAN J. HOFFMAN $\ddagger$, AND WALTER KERN ${ }^{\dagger}$
}

\begin{abstract}
Given an ordering of the variables according to nonincreasing coefficients of the objective function $c^{T} x$, the nonnegative matrix $A$ is said to be greedy if, under arbitrary nonnegative constraint vectors $b$ and $h$, the greedy algorithm maximizes $c^{T} x$ subject to $A x \leq b, 0 \leq x \leq h$. Extending a result of Hoffman, Kolen, and Sakarovitch for $(0,1)$-matrices, we characterize greedy matrices in terms of forbidden submatrices, which yields polynomial recognition algorithms for various classes of greedy matrices. The general recognition problem for the existence of forbidden submatrices is shown to be NP-complete
\end{abstract}

Key words. greedy algorithm, linear program

AMS subject classification. $90 \mathrm{C} 27$

1. Introduction. The greedy algorithm constitutes a fundamental algorithmic principle in combinatorics and has received considerable attention within the framework of linear programming. Basically, one orders the components of the objective function according to nonincreasing values and then constructs a feasible solution for the linear program greedily in that order of the variables. The problem is to find out under what conditions the greedy solution is optimal.

A well-known example is the (generalization of the) matroid greedy algorithm of Edmonds (1970), which refers to $(0,1)$-constraint matrices $A$ and is robust relative to arbitrary linear objective functions as long as the vector $b$ in the feasibility conditions

$$
A x \leq b, \quad x \geq 0
$$

corresponds to a submodular set function.

A different line of research on greedily solvable linear programs was initiated by Hoffman, Kolen, and Sakarovitch (1985). Here the order of the variables is fixed; i.e., one only considers objective functions $c^{T} x$, where $c^{T}=\left(c_{1}, \ldots, c_{n}\right)$ satisfies $c_{1} \geq c_{2} \geq$ $\cdots \geq c_{n}$. The matrix $A$ is then termed (box)-greedy if the greedy algorithm successfully solves the linear program

$$
\max c^{T} x \text { s.t. } A x \leq b, 0 \leq x \leq h,
$$

relative to any feasible choice of $c, b$, and $h$. Hoffman, Kolen, and Sakarovitch show that greedy $(0,1)$-matrices $A$ can be characterized by two simple forbidden submatrices.

It is our purpose here to provide a combinatorial characterization of arbitrary nonnegative greedy matrices that was announced (without proof) in Hoffman (1985). We do this in $\S 2$ by showing that it suffices to check greediness for the quasi-diagonal submatrices of $A$, i.e., for those submatrices that can be brought into essentially

\footnotetext{
$\dagger$ Department of Applied Mathematics, University of Twente, P.O. Box 217, 7500 AE Enschede, The Netherlands (faigle@math.utwente.nl and kern@math.utwente.nl).

$\ddagger$ Department of Mathematical Sciences, IBM Research Division, T. J. Watson Research Center, P.O. Box 218, Yorktown Heights, NY 10598 (hoffa@watson.ibm.com).
}

* Received by the editors November 23, 1993; accepted for publication (in revised form) January $27,1995$. 
diagonal form by a suitable permutation of the rows. Checking greediness of particular quasi-diagonal submatrices turns out to be very simple.

Our characterization yields polynomial recognition algorithms for various special classes of nonnegative box-greedy matrices (cf. §3). The general problem turns out to be NP-complete.

2. Greedy matrices. Let $A \in \mathbb{R}_{+}^{m \times n}$ be a nonnegative real matrix and $b \in$ $\mathbb{R}_{+}^{m}, h \in \mathbb{R}_{+}^{n}$ be nonnegative vectors. The greedy algorithm constructs a feasible solution $x^{*}=\left(x_{1}^{*}, \ldots, x_{n}^{*}\right)^{T} \in \mathbb{R}^{n}$ for the system

$$
A x \leq b, \quad 0 \leq x \leq h
$$

as follows:

$$
\begin{aligned}
\text { FOR } k & =1, \ldots, n \\
x_{k}^{*} & =\max \left\{x \in \mathbb{R} \mid\left(x_{1}^{*}, \ldots, x_{k-1}^{*}, x, 0, \ldots, 0\right)^{T} \text { feasible }\right\} .
\end{aligned}
$$

We say that the matrix $A$ is (box)-greedy if, for any choice of $b$ and $h$, the greedy solution is optimal for the linear program

$$
\max c^{T} x \text { s.t. } A x \leq b, 0 \leq x \leq h,
$$

whenever $c=\left(c_{1}, \ldots, c_{n}\right)^{T}$ satisfies $c_{1} \geq c_{2} \geq \cdots \geq c_{n} \geq 0$. Note that the greedy algorithm for (2.2) depends on $b$ and $h$ but not on $c$. Our aim is to characterize greedy matrices in terms of inherent combinatorial properties without any reference to constraint vectors $b$ and $h$. Choosing $b$ and $h$ appropriately, it is easy to see that the matrix $A$ can only be greedy if all submatrices of $A$ are greedy. We are interested in particular submatrices of $A$.

The matrix $B$ is a normalized submatrix of $A$ if there is a submatrix $A^{\prime}$ of $A$ such that the first element in each row of $A^{\prime}$ is strictly positive and $B$ arises from $A^{\prime}$ by dividing each row by its first element. Let $B_{0}$ be the submatrix obtained by removing the first column of $B$. So

$$
B=\left[\mathbf{1} \mid B_{0}\right],
$$

where 1 denotes the vector with all 1's of appropriate dimension.

The normalized submatrix $B$ of $A$ is quasi-diagonal if the rows of $B_{0}$ can be permuted so that a diagonal matrix results. With each quasi-diagonal normalized submatrix $B$ we associate the weight

$$
w(B)=\sum \frac{1}{d_{j}}
$$

where the $d_{j}$ 's are the nonzero entries of $B_{0}$. (Set $w(B)=0$ if $B_{0}$ contains no nonzero element.)

The quasi-diagonal submatrix $B$ is feasible if $w(B) \leq 1$.

LEMMA. If $A$ is greedy, then every quasi-diagonal normalized submatrix $B$ is feasible.

Proof. Suppose $B$ is normalized submatrix with $w(B)>1$, where without loss of generality $B_{0}=\operatorname{diag}\left(d_{1}, \ldots, d_{k}\right)$ and $d_{1} d_{2} \ldots d_{k} \neq 0$.

Then the greedy algorithm yields objective function value 1 for the linear program

$$
\max 1^{T} x \text { s.t. } B x \leq \mathbf{1}, x \geq 0 .
$$


Since $\bar{x}=\left(0, d_{1}^{-1}, \ldots, d_{k}^{-1}\right)$ is feasible with $\mathbf{1}^{T} x=w(B)>1, B$ is obviously not greedy. Hence also $A$ is not greedy.

We now state a sufficient condition.

Proposition. Assume that for every normalized submatrix $B$ of $A$, the linear inequality system

$$
B_{0} \lambda \geq \mathbf{1}, \quad \lambda \geq 0
$$

either is not feasible or has a solution $\lambda$ with $\mathbf{1}^{T} \lambda \leq 1$. Then $A$ is greedy.

Proof. Consider the feasible linear program

$$
\max c^{T} x \text { s.t. } A x \leq b, 0 \leq x \leq h,
$$

with $c_{1} \geq \cdots \geq c_{n} \geq 0$. Among all optimal solutions for the linear program, let $\bar{x}=\left(\bar{x}_{1}, \ldots, \bar{x}_{n}\right)^{T}$ be one with first component $\bar{x}_{1}$ as large as possible.

If $\bar{x}_{1}=x_{1}^{*}$, where $x^{*}=\left(x_{1}^{*}, \ldots, x_{n}^{*}\right)$ is the greedy solution, we may assume by induction on the number of variables that

$$
c_{2} \bar{x}_{2}+\cdots+c_{n} \bar{x}_{n} \leq c_{2} x_{2}^{*}+\cdots+c_{n} x_{n}^{*}
$$

and hence $c^{T} x^{*} \geq c^{T} \bar{x}$, i.e., that $x^{*}$ is optimal.

So suppose $\bar{x}_{1}<x_{1}^{*}$. We will then exhibit a feasible solution $\tilde{x}=\left(\tilde{x}_{1}, \ldots, \tilde{x}_{n}\right)$ with $\tilde{x}_{1}>\bar{x}_{1}$ and $c^{T} \tilde{x} \geq c^{T} \bar{x}$, a contradiction to the choice of $\bar{x}$.

Let $A^{\prime}$ be the submatrix of those rows $A_{i}$ of $A$ with leading coefficient $a_{i 1} \neq 0$ and $A_{i} \bar{x}=b_{i}$, restricted to those columns $A^{j}$ such that $j=1$ or $\bar{x}_{j}>0$. Denote by $B$ the normalized submatrix associated with $A^{\prime}$.

Because $\bar{x}_{1}<x_{1}^{*}$, the matrix $B_{0}$ has (at least) one nonzero element in each row. Hence $B_{0} \lambda \geq \mathbf{1}, \lambda \geq 0$, is feasible and we may assume that $\mathbf{1}^{T} \lambda \leq 1$ holds.

Let $\varepsilon_{1}>0$ be such that the vector $\left(\bar{x}_{1}+\varepsilon_{1}, \bar{x}_{2}, \ldots, \bar{x}_{n}\right)$ does not violate any constraint $A_{i}$ outside $A^{\prime}$, and choose $\varepsilon_{2}>0$ such that

$$
\bar{x}_{j}-\varepsilon_{2} \lambda_{j} \geq 0 \text { for all } \bar{x}_{j}>0, j \geq 2 .
$$

With $\varepsilon=\min \left\{\varepsilon_{1}, \varepsilon_{2}\right\}>0$, we thus conclude from $B_{0} \lambda \geq \mathbf{1}$ that the vector $\tilde{x}=$ $\left(\tilde{x}_{1}, \ldots, \tilde{x}_{n}\right)^{T}$ given by

$$
\tilde{x}_{j}=\left\{\begin{array}{cl}
\bar{x}_{1}+\varepsilon & \text { if } j=1 \\
\bar{x}_{j}-\varepsilon \lambda_{j} & \text { if } j \geq 2 \text { and } \bar{x}_{j}>0 \\
0 & \text { otherwise }
\end{array}\right.
$$

is feasible for the linear program. Moreover, $c_{1} \geq \max \left\{c_{j} \mid j \geq 2\right\}$ and $\mathbf{1}^{T} \lambda \leq 1$ yield $c^{T} \tilde{x} \geq c^{T} \bar{x}$, which is the desired contradiction.

Our main result asserts that the necessary condition in the lemma is equivalent with the sufficient condition in the proposition.

TheOREM 1. Let $A$ be a nonnegative matrix. Then $A$ is greedy if and only if every quasi-diagonal normalized submatrix $B$ of $A$ is feasible.

Proof. We show that $A$ violates the necessary condition of the lemma if $A$ violates the sufficient condition of the proposition.

Suppose that $B$ is a (row and column) minimal normalized submatrix of $A$ such that the linear program

$$
\min \mathbf{1}^{T} \lambda \text { s.t. } B_{0} \lambda \geq \mathbf{1}, \lambda \geq 0
$$


is feasible with optimal objective function value $z_{0}>1$. We claim that $B$ contains some infeasible quasi-diagonal submatrix.

To fix notation, let $E$ be the index set of the rows of $B_{0}$ and $E_{1}, \ldots, E_{k}$ be the supports of the $k$ columns of $B_{0}$. Because $B_{0}$ was chosen feasible and column-minimal with objective function value strictly greater than 1 , we must have

$$
\begin{gathered}
E \subseteq \bigcup_{s=1}^{k} E_{s} \\
\text { and } E \nsubseteq \bigcup_{s \neq t} E_{s} \text { for } t=1, \ldots, k .
\end{gathered}
$$

So there are elements $e_{1}, \ldots, e_{k} \in E$ with $e_{s} \in E_{s}$ and $e_{s} \notin E_{t}$ for $t \neq s$. Now restrict $B$ to the submatrix $\bar{B}$ consisting of the rows of $B$ that correspond to $\left\{e_{1}, \ldots, e_{k}\right\}$, and observe that $\bar{B}$ is a quasi-diagonal normalized submatrix of $A$. If $d_{1}, \ldots, d_{k}$ are the nonzero entries of $\bar{B}_{0}$, the proof is finished once we can establish the relation

$$
\frac{1}{d_{1}}+\cdots+\frac{1}{d_{k}}=z_{0}>1
$$

Let $\bar{\lambda}=\left(\bar{\lambda}_{1}, \ldots, \bar{\lambda}_{k}\right)^{T}$ be an optimal feasible solution for (2.4). Then $\bar{B}_{0} \bar{\lambda} \geq \mathbf{1}$ implies

$$
\bar{\lambda}_{s} \geq \frac{1}{d_{s}} \text { for } s=1, \ldots, k \text {. }
$$

If $\bar{\lambda}_{1} \neq d_{\underline{1}}^{-1}$, for example, then the $e_{1}$-constraint in (2.4) is not binding for the optimal solution $\bar{\lambda}$. Hence also the linear program

$$
\min \mathbf{1}^{T} \lambda \text { s.t. } B_{0}^{\prime} \lambda \geq \mathbf{1}, \lambda \geq 0,
$$

where $\left(2.4^{\prime}\right)$ is obtained by dropping the $e_{1}$-constraint in $(2.4)$, is feasible with optimal objective function value $z_{0}^{\prime}>1$, which contradicts the row-minimality of the ma$\operatorname{trix} B$.

Hence we conclude $\bar{\lambda}=\left(d_{1}^{-1}, \ldots, d_{k}^{-1}\right)^{T}$.

3. Recognition complexity of box-greedy matrices. For the fixed parameter $K \geq 0$, let $\mathcal{M}(K)$ be the class of all nonnegative matrices $A$ such that the maximum ratio of every pair of nonzero elements in each row of $A$ is bounded by $K$.

CoRollary 1. For every fixed $K \geq 0$, there is a polynomial algorithm to decide whether the matrix $A \in \mathcal{M}(K)$ is (box-)greedy.

Because $A \in \mathcal{M}(K)$, we know that the positive entries in each quasi-diagonal normalized submatrix $B$ lie in the interval $[1, K]$. Hence we have $w(B)>1$, whenever $B_{0}$ has more than $K$ nonzero elements. So it suffices to check the condition of Theorem 1 for quasi-diagonal normalized submatrices with at most $K+1$ rows and $K+2$ columns, which can be done in polynomial time for fixed $K$.

A further specialization yields the characterization of greedy $(0,1)$-matrices as "totally balanced" matrices due to Hoffman, Kolen, and Sakarovitch (1985).

Corollary 2. Let $A$ be a $(0,1)$-matrix. Then $A$ is greedy if and only if neither of the following two matrices occurs as a submatrix:

$$
\left(\begin{array}{lll}
1 & 1 & 0 \\
1 & 0 & 1
\end{array}\right), \quad\left(\begin{array}{lll}
1 & 0 & 1 \\
1 & 1 & 0
\end{array}\right)
$$


Reasoning similarly as in Corollary 1 , it is also easy to recognize nonnegative greedy matrices $A$ with arbitrary coefficients if we bound the number of rows or the number of columns. Let us turn to the recognition complexity for general greedy matrices. To be more precise, consider the following decision problem.

Instance. A nonnegative matrix $A \in \mathbb{Q}^{m \times n}$.

Question. Does $A$ contain an infeasible quasi-diagonal normalized submatrix?

THEOREM 2. The decision problem above is NP-complete.

Proof. Since infeasibility of a given quasi-diagonal submatrix can be checked in polynomial time, the decision problem is in NP. To prove that it is NP-complete it suffices to show that it is at least as hard as the problem of deciding whether a graph $G$ with $n$ nodes has a stable set of size $k+1$ (cf. Garey and Johnson (1979)).

We associate with $G$ the $(n \times n)$-matrix $A_{0}=\left(a_{i j}\right)$, where

$$
a_{i j}=\left\{\begin{array}{cl}
n^{j+1} & \text { if } i<j \text { and } i j \text { is an edge in } G \\
k+\frac{1}{2} & \text { if } i=j \\
0 & \text { otherwise }
\end{array}\right.
$$

Assuming $n>k \geq 2$, consider now the matrix $A=\left[\mathbf{1} \mid A_{0}\right]$. Clearly, if $G$ contains a stable set of size $k+1, A$ contains an infeasible quasi-diagonal submatrix $B=\left[1 \mid B_{0}\right]$, where the nonzero elements of $B_{0}$ form a subset of the diagonal of $A_{0}$.

Conversely, let $B=\left[\mathbf{1} \mid B_{0}\right]$ be a normalized quasi-diagonal submatrix of $A$. Suppose that $G$ has no stable set of size $k+1$. We claim $w(B) \leq 1$. To verify the claim, we distinguish two cases.

If $B$ is a normalized submatrix of the submatrix $A_{0}$, then every nonzero entry of $B_{0}$ has size at least $n$. So $w(B) \leq n \cdot(1 / n)=1$.

In the other case, $B_{0}$ is a submatrix of $A_{0}$. Because $G$ has no stable set of size $k+1, B_{0}$ contains at most $k$ elements from the diagonal of $A_{0}$. Hence

$$
w(B) \leq \frac{k}{k+\frac{1}{2}}+\sum_{j=1}^{n} \frac{1}{n^{j+1}} \leq \frac{2 k}{2 k+1}+\frac{1}{n^{2}-n} \leq 1
$$

4. Remarks and open problems. While Theorem 1 characterizes nonnegative matrices $A$ that are greedy relative to any box constraints $0 \leq x \leq h$, it is interesting to know the structure of general "greedy" matrices. Hoffman (1992) gives a characterization of $(0,1)$-matrices $A$ for which the greedy algorithm solves the linear program

$$
\max \mathbf{1}^{T} x \text { s.t. } A x \leq b, x \geq 0
$$

and its dual relative to any $b$. An extension of this result to arbitrary nonnegative matrices is open.

We finally mention a related problem. If $x^{*}$ is the greedy solution to the nonnegative linear program

$$
\max c^{T} x \text { s.t. } A x \leq b,
$$

it is not hard to see that $x^{*}$ is a basic solution. Is there a "simple" way to decide whether $x^{*}$ is optimal? For example, if $x^{*}$ is nondegenerate, it suffices to consider the basis $B$ associated with $x^{*}$ and to check whether $y=c^{T} B^{-1}$ is nonnegative, i.e., whether the vector $c^{T} B^{-1}$ yields a dually feasible solution (cf. Kovalev and Vasilkov (1993)). 


\section{REFERENCES}

J. Edmonds (1970), Submodular functions, matroids and certain polyhedra, in Combinatorial Structures and Their Applications, R. Guy et al., eds., Gordon and Breach, New York, pp. 69-87.

M. R. GAREY AND D. S. Johnson (1979), Computers and Intractability: A Guide to the Theory of NP-Completeness, Freeman, San Francisco, CA.

A. J. Hoffman (1985), On greedy algorithms that succeed, in Surveys in Combinatorics, I. Anderson, ed., Cambridge University Press, Cambridge, pp. 97-112.

A. J. HofFMAN (1992), On simple combinatorial optimization problems, Discrete Math., 106/107, pp. 285-289.

A. J. Hoffman, A. W. J. Kolen, And M. SAKarovitch (1985), Totally-balanced and greedy matrices, SIAM J. Alg. Discrete Methods, 6, pp. 721-730.

M. M. KOVAlEV AND D. M. VASILKOV (1993), The canonical order and optimization problems, preprint, Fac. of Applied Mathematics, Belarusian State University, Minsk; Z. Oper. Res., 1996. 